\title{
STUDY OF PHOTOELECTRON EMISSION YIELD FROM LAYERED STRUCTURES IN PRESENCE OF RESONANCE-ENHANCED X-RAY PROPAGATION EFFECT
}

\author{
J.B. PELKa \\ Institute of Physics, Polish Academy of Sciences \\ Al. Lotników 32/46, 02-668 Warsaw, Poland \\ S. Lagomarsino, A. Cedola \\ Istituto dello Stato Solido CNR, V. Cineto Romano 42, Roma, Italy \\ S. Di FONZo AND W. JARK \\ Sincrotrone Trieste, Padriciano 99, 34012 Trieste, Italy
}

In this work we present the new experimental results of total photoelectric yield as well as energy distribution of photoelectrons excited in a thin carbon film deposited on $\mathrm{Ni}$ mirror in the presence of resonance-enhanced $\mathrm{X}$-ray propagation effect. The measurements were performed using conventional X-ray tube as a radiation source for the energy $\mathrm{Cu} K_{\alpha}(8047 \mathrm{keV})$. The spectra were recorded using a flow proportional electron counter with energy resolution of about $15 \%$, and multichannel pulse height analyzer. A comparison with the reflectivity spectra recorded at the same time show an excellent correlation of both kinds of spectra, consistently with the theoretical prediction. A map of electron energy distribution is reported. Although the applied electron counter was of low energetic resolution the recorded spectra show characteristic regularities and indicate that the photoelectron yield excited in the presence of resonance-enhanced X-ray propagation effect can provide depth dependent information about impurity distribution and processes in thin layers.

PACS numbers: 68.65.+g, 78.20.-e 


\section{Introduction}

The resonance-enhanced propagation of X-rays (denoted here as REXPE) in thin films deposited on an X-ray reflecting mirror was anticipated theoretically in 1991 by Wang et al. [1] and also by de Boer [2]. A year later the effect was experimentally confirmed in an organic thin film deposited on gold mirror by Wang, Bedzyk and Caffrey, who used a synchrotron radiation from hard X-ray wiggler [3]. Recently, the resonant effects have been found also in thin carbon layers using the conventional X-ray tubes as radiation sources $[4,5]$. The effect of resonance observed in these experiments strongly enhances the electric field inside the film, forming the X-ray standing waves (XSW) of a variable period, depending on the angle of incidence of the primary beam. The amplitudes of the field exceed up to two orders of magnitude the XSW amplitudes observed in the layers without resonant conditions.

The conditions for the REXPE are fulfilled for a particular combination of layer thickness, its electron density (with respect to deeper neighboring layer), and wavelength of the excited X-ray beam. When the monochromatic X-ray beam of small divergence impinges on solid surface under a critical angle, $\theta_{c}$, which depends on the wavelength and on the nature of the reflecting surface, it is totally reflected [1]. Due to the interference between the incoming and the reflected beams, a standing wave field is formed above the surface with a periodicity $D$ that depends on the incident angle and on the wavelength, $D=\lambda /(2 \sin \theta)$. The $\theta_{c}$ is equal to $\sqrt{2 \delta}$, where $1-\delta$ is the real part of the refractive index for the material. The total external reflection region under this critical angle is very small. This is because the $\delta$ values are in the $\mathrm{X}$-ray wavelength region for all materials of the order $10^{-4}-10^{-6}$. If a thin film is present on the reflecting surface, formed from the material characterized by $\theta_{\mathrm{c} 2}>\theta_{\mathrm{c} 1}$, the incoming beam penetrates easily through this layer, where a refraction effect occurs, but it is totally reflected by the underlying mirror (see Fig. 1A). This phenomenon can take place within the angles of incidence between $\theta_{\mathrm{c} 1}$ and $\theta_{\mathrm{c} 2}$. Consequently, a standing wave field is formed inside the top layer with the standing wave nodes on its both surfaces. Under these conditions, a constructive interference takes place, with a considerable enhancement of the $E$-field intensity in the layer. The resonant enhancement of the $E$-field only occurs when the coating material has an electron density lower than that of the reflecting surface.

Both for the single layer, and for a more complex system composed of several layers the reflectivity and $E$-field distribution in the sample can be calculated from the same basic Fresnel equation, which can be applied recursively starting from the substrate towards the top interface [1]. In Fig. 1B theoretical $E$-field calculations are shown for a sample construction that was used in the present study: $98.5 \mathrm{~nm}$ thick $\mathrm{C}$ film on a $82 \mathrm{~nm} \mathrm{Ni}$ reflecting layer. A $0.5 \mathrm{~nm}$ Ti ultra-thin layer deposited exactly in the center of the C film was introduced as a marker to monitor the $E$-field intensity distribution. While increasing the angle of incidence, we can observe several orders of resonance. Comparing the ordinary XSW intensities observed in the case of a simple total reflection, above the mirror surface, the intensity of the $E$-field in the first order can reach values of one order of magnitude higher or 


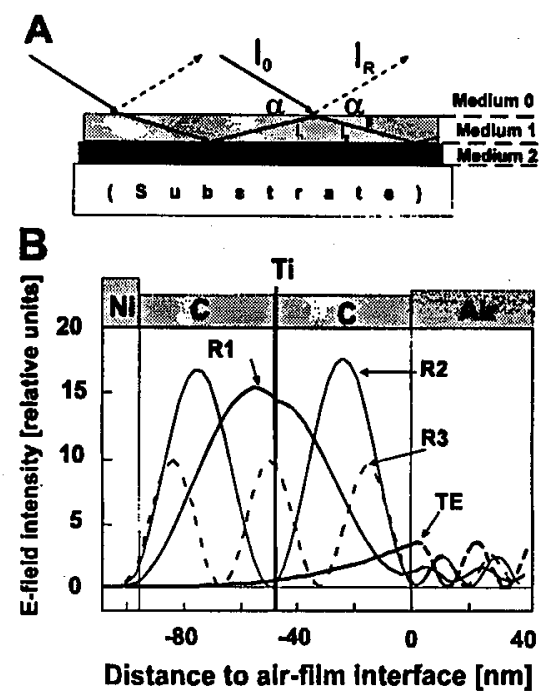

Fig. 1. (A) Sketch of the X-ray beam interaction with the thin film within the region of resonance. Medium 0 is air (vacuum). If medium 1 is lighter than medium 2, the condition for enhancement of SW $E$-field in the layer for some angles can occur; (B) Calculated distribution of the $E$-field intensity for the sample studied in this work. The calculations were performed assuming various angles of incidence, corresponding to the positions of resonances: R1 - the 1st, R2 - the 2nd, R3 - the 3rd; and also for the angle below the critical one for carbon layer (TE).

even more higher than in the case of REXPE. Our calculations show it clearly, when comparing the intensities of the $E$-field above the top surface in the air, and in the $\mathrm{C}$ layer (see Fig. 1B). The Ti layer, although very thin $(0.5 \mathrm{~nm})$, modifies significantly the $E$-field intensity. The calculations show that the Ti layer suppresses the $E$-field intensity in the 1st order by the factor of 2 . The suppression for higher orders is not so high.

In our previous papers it was shown that the yield of secondary radiation, like fluorescence or photoelectrons obtained with $\mathrm{Cr} K_{\alpha}, \mathrm{Cu} K_{\alpha}$ and Mo $K_{\alpha}$ radiation from conventional X-ray tubes $[4,5]$, which is proportional to the $E$-field intensity can be an efficient probe enabling a study of impurity distribution and some other properties or processes in the wave guide sample. The spectra were found to be free of background coming from any structure that is under the totally reflected mirror layer. The background from the mirror itself can be only due to penetration by the evanescent wave, of very low intensity. The preliminary results of total electron yield reported previously did not provide however any information about photoelectron energy distribution. In the present paper we give such information for the first time. 


\section{Experimental}

The sample used in this study was of the construction described above. It was produced by sputtering deposition on the glass substrate in the multilayer laboratory of the Sincrotrone Trieste. Sputtering was achieved by the use of a triode assisted and magnetically confined plasma in a low pressure $\mathrm{Ar}$ atmosphere $(p=0.1 \mathrm{~Pa})$. Therefore, there is an amount of Ar impurity of uniform distribution in the sample, as it was confirmed by the measurements of Ar fluorescence yield. The detailed description of sample production and tests can be found elsewhere [4]. The roughness of the sample top surface and all interfaces was estimated to be about $0.7 \mathrm{~nm}$ rms. The same sample was used to study the fluorescence yield excited with $\mathrm{Cr} K_{\alpha}$ radiation, which were published earlier [4, 5].

The photoelectron yield was recorded with the $\mathrm{Cu} K_{\alpha}$ radiation at the Institute of Physics, Polish Academy of Sciences, Warsaw, by double-crystal spectrometer equipped with a flow proportional camera as a detector of photoelectrons [6]. The photoelectron spectra were collected in a pulse height analyzer (PHA) device and then saved in computer memory separately for each measuring point. The reflectivity of the sample was recorded at the same time.

\section{Results and discussion}

The results of the total photoelectron yield and reflectivity are shown in Fig. 2 (lower part). No corrections were applied to the experimental results. A comparison of the reflectivity with the theoretical calculation smeared due to finite beam divergence showed an excellent agreement and is not shown here. The electron energy distribution of the spectra is shown in Fig. 2 (upper part) in a form of a map with respect to angular position of the incident beam ( $x$ axis), and PHA channel number ( $y$ axis). The position of zero energy channel is -15 , as the PHA base channel was set to this value to cut the noise.

The detailed analysis of total photoelectron yield spectra as well as the associated reflectivity has been done elsewhere $[5,7]$. Therefore, in this paper we will concentrate on the analysis of the measured electron encrgy distribution. Let us remind here only that the angular positions of resonances are indicated by the angular positions of reflectivity minima, and in the case regarded here we can easily find seven such positions, which are related to the first, second, etc. orders of resonant XSW field in the sample. At angles steeper than the critical one for the $\mathrm{C} / \mathrm{Ni}$ interface the radiation penetrates the $\mathrm{Ni}$ mirror, and there are no more conditions for resonance.

The flow proportional counter registers both photoelectrons and Auger electrons emitted from the sample. The detailed electron scattering processes cause a complicated depth profile for electrons escaping from the solid with a particular energy loss. Only a very low fraction of all electrons can escape from the solid without any energy loss. Despite the complexity of the scattering processes the electrons undergo in the layer, the observed experimental angular dependence of the total electron yield is clearly related to the reflectivity spectrum. Within the region of resonance, its shape looks like the mirror image of the reflectivity, repeating this way the relative intensities of the reflectivity minima (as the photoelectric 

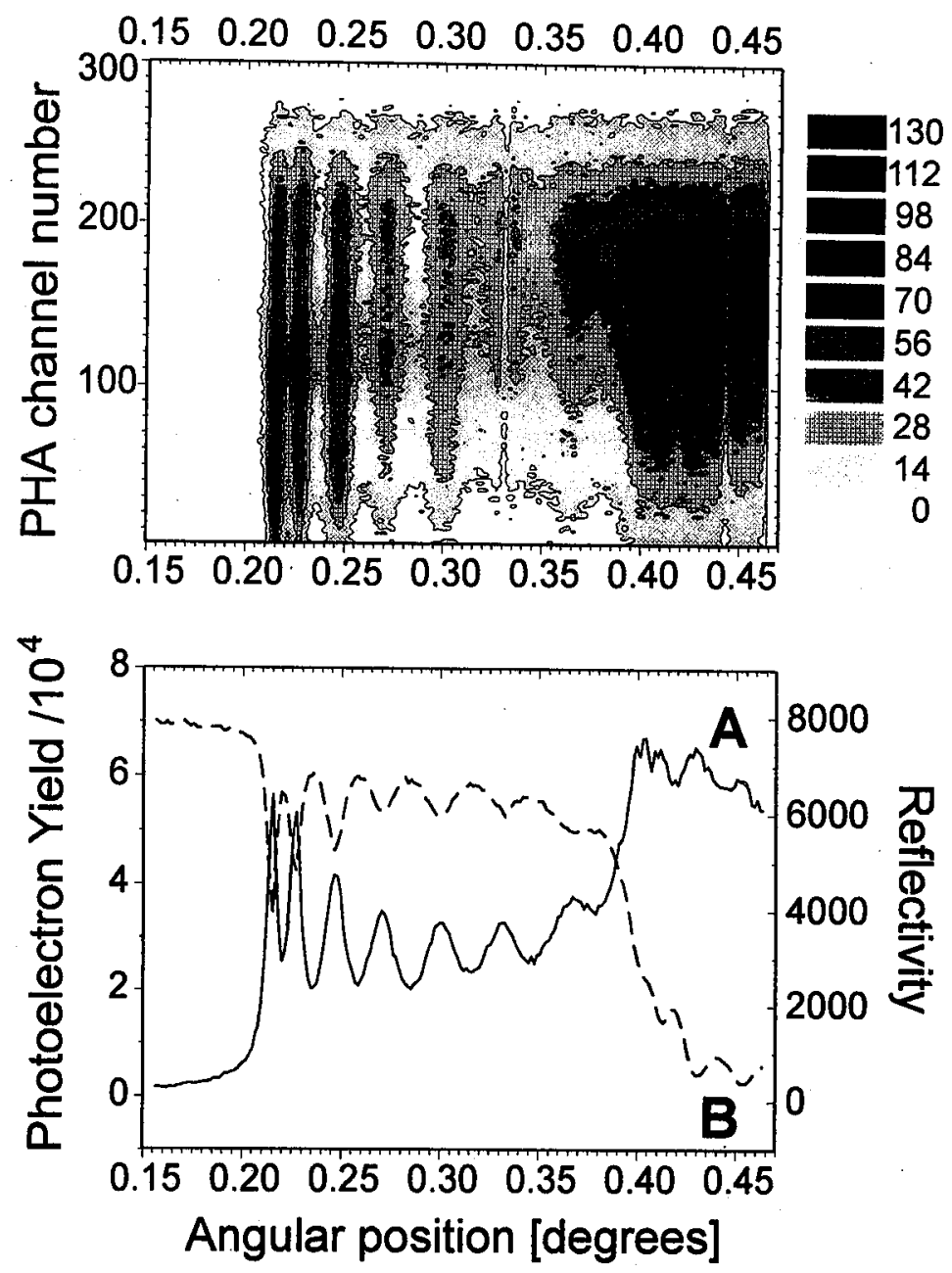

Fig. 2. Upper part: map of measured electron energy distribution from the sample with respect to angular position of the incident beam ( $x$ axis), and PHA channel number ( $y$ axis); Lower part: A - Total photoelectron yield (solid line) and B - reflectivity (dashed line) from the sample in a function of incident beam angular position.

maxima). At higher angles, the measured photoelectron yield, after having reached the local maximal value decreases slowly with some modulation, vanishing with angle.

In the electron energy distribution map shown in Fig. 2 the same structure of the maxima and minima as a function of angular position is evident. We can distinguish the seven regions of resonance, which are increasing in width with an increase in angle. The width of the electron energy distribution for lower resonances is broader comparing to the higher ones. Above the angle of about $0.38^{\circ}$ the energy map changes; it reflects passing over the critical angle for $\mathrm{C} / \mathrm{Ni}$ interface, 
and penetration of X-rays into the $\mathrm{Ni}$ layer. The energy distribution, which at resonant positions seems to be structured, or multipeak, here changes to a single peak, with maximum around channel No. 160 .

From the spectra collected at angular positions below the critical angle of carbon layer we were able to calibrate the energy width of the PIIA channel and the $y$ scale of the map. This followed the fact that photoelectrons at angles below the critical one are produced in a very thin layer only by an evanescent wave, and we can assume that they do not undergo any significant energy losses in a sample. We could expect here the photoelectrons from $\mathrm{C}$ and Ar atoms, which constitute the top layer near surface, and also from some atoms adsorbed at sample surface, e.g. O. From binding energies for the atoms [8] and positions of three peaks well defined in these spectra we obtained the channel energetic width of about $27.87 \mathrm{eV}$ and linearity of the scale better than $1 \%$, what is a surprisingly good result. The carbon $\mathrm{K}$ peak (b.e. $284 \mathrm{eV}, E_{\text {kin }}$ about $7760 \mathrm{eV}$ ) was ascribed to the channel 263 and the Ar K peak (b.e. $3205 \mathrm{eV}, E_{\text {kin }}$ about $4840 \mathrm{eV}$ ) to the channel 159.

In the spectra mapped in Fig. 2 we can distinguish some structures, which change with angle in a regular way. The spectra at angular positions of reflectivity maxima are weaker comparing to the neighboring positions of minima; it is due to the fact that there is no resonance at these positions and no $E$-field enhancement inside the layer occurs. Therefore the contribution of electrons from near-surface parts of the layer is higher for the non-resonant positions. It results in the spread of the spectra toward higher energy channels, and in a relative increase in intensity in the central part, around channel 160 . The structure of the spectra at positions of the first five resonances, are different. In the first resonance we can distinguish three peaks: the first one around channel No. 50, the second of the highest intensity, around channel No. 100, and the last one, around channel No. 180. The first peak is well pronounced at odd resonant positions, with the maximum at the same energy (around channel No. 50). It seems to disappear at positions of even resonances. Contrary to a stable position of the first peak, the maxima of the two others shift towards higher energies as the angle increases, up to the position of the fifth resonance. Intensities of the peaks decrease with order of resonance. For the angular positions above the last (seventh) resonance, the spectra are composed of one broad peak, and their intensity first rapidly increases, and then slowly decreases.

The quantitative analysis of the observed features exceeds the scope of the present paper and will be published later. It is possible however to give a qualitative interpretation of the recorded spectra within the region of resonances. The second and third peak observed at resonant positions can be identified as produced by photoelectrons excited, respectively, from $\mathrm{Ar}$ and $\mathrm{C}$ atoms and shifted towards lower energy. This shift is due to the energy losses the electrons undergo on their path from the inside of the layer to the surface. The shift of these maxima toward higher energies with an increase in order of resonance can be explained when we will take into account that the higher is the order of resonance, the closer to the top surface is an antinode of $E$-field. Because all antinodes in the $E$-field distribution at a given resonance are of approximately the same amplitude (see. Fig. 1B), the electrons excited in the nearest node will dominate in energy and intensity 
over contributions from other nodes. The observed behavior of the first maximum suggests that it is contributed by the photoelectrons excited in the central part of the layer. The appearing of the peak in odd resonances, where there always is an antinode of the $E$-field, and disappearing in even resonances, where a node of $E$-field exists, together with its stable position, clearly explain it. It suggests that this maximum is formed by photoelectrons excited in the ultra-thin Ti marker layer. The photoelectrons excited in Ni sublayer dominate in the spectra above the position of the last resonance. However, the single-peak form of the spectra appears already earlier - it is evident from the sixth resonance. It can be explained by the influence of the photoelectrons excited by the evanescent wave penetrating the $\mathrm{Ni}$ mirror.

\section{Conclusions}

The unique properties of the resonance-enhanced propagation of X-rays in thin films allow for the efficient applications of this phenomenon to the studies of structure and various processes in thin layers. In this paper we reported the study of photoelectron spectra excited in thin layer under REXPE conditions. The analysis of recorded energy distributions of the photoelectrons allowed for identification of observed main peaks and for qualitative interpretation of the spectra. The obtained results are consistent with the theoretical model of REXPE angular and in-depth dependent $E$-field distribution. $A$ more precise quantitative interpretation of the results needs still some theoretical effort in order to include the parameters characterizing the photoelectron interactions in the sample, and it will be published later.

\section{Acknowledgments}

This work was supported in part by the Committee for Scientific Research, grant No. 2P03B 02109.

\section{References}

[1] J. Wang, M.J. Bedzyk, T.L. Penner, M. Caffrey, Nature 354, 377 (1991).

[2] D.K.G. De Boer, Phys. Rev. B 44, 498 (1991).

[3] J. Wang, M.J. Bedzyk, M. Caffrey, Science 258, 775 (1992).

[4] S. Di Fonzo, W. Jark, S. Lagomarsino, A. Cedola, B. Muller, J.B. Pełka, Thin Solid Films 287, 288 (1996).

[5] J.B. Pełka, S. Lagomarsino, A. Cedola, S. Di Fonzo, W. Jark, B. Muller, J. Domagala, in: Zastosowanie Promieniowania Synchrotronowego, Ed. E. Sobczak, Wyd. Fundacji im. W. Świętosławskiego, Warszawa 1995, p. 130 (in Polish).

[6] J. Auleytner, K. Godwod, J.B. Pełka, Cryst. Res. Technol. 30, 483 (1995).

[7] J.B. Pełka, S. Lagomarsino, W. Jark, J. Domagala, Acta Phys. Pol. A 89, 323 (1996).

[8] Practical Surface Analysis by Auger and X-ray Photoelectron Spectroscopy, Eds. D. Briggs, M.P. Seah, Wiley\&Sons, Chichester 1987, p. 484. 